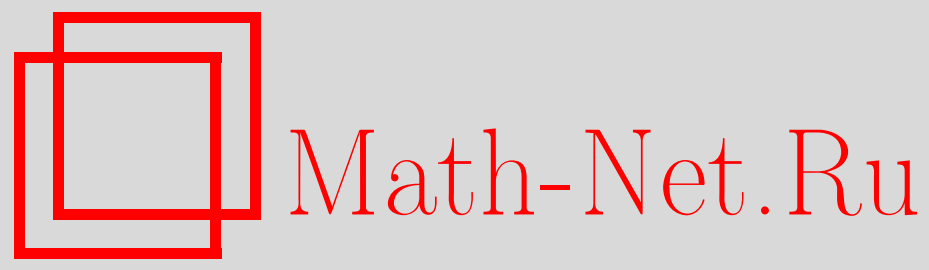

А. Ю. Окуньков, Замечание о полиноме Гильберта сферического многообразия, Функи. анализ и его прил., 1997, том 31, выпуск 2, 82-85

DOI: https://doi.org/10.4213/faa466

Использование Общероссийского математического портала MathNet.Ru подразумевает, что вы прочитали и согласны с пользовательским соглашением

http://www . mathnet.ru/rus/agreement

Параметры загрузки:

IP : 18.208 .226 .222

26 апреля 2023 г., 18:25:38

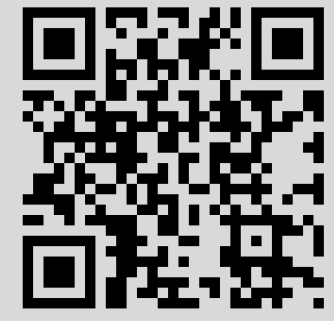


(б) Если $\Delta(\bar{A})$ плотно в $A_{0}$ и $A_{1} u \overrightarrow{1} \leqslant \vec{q}<\vec{\infty}, 0<\theta<1$, mо $\left(A_{0}, A_{1}\right)_{\theta \vec{q}}^{*}=$ $\left(A_{0}^{*}, A_{1}^{*}\right)_{\theta \vec{q}^{\prime}}$, где $1 / q_{i}+1 / q_{i}^{\prime}=1, i=1, \ldots, n$.

Теорема 5 (о реитерации). Пусть $\bar{A}=\left(A_{0}, A_{1}\right)$ - совместимал банахова nара, $\vec{q}_{0}, \vec{q}_{1}, \vec{d}-$ допустимье векторьи, $r=\min \left\{i: q_{i}^{0} \neq q_{i}^{1}, i=1, \ldots, n\right\}$. Тогда при $\vec{q}=\left(q_{1}^{0}, \ldots, q_{r-1}^{0}, q_{r}, d_{1}, \ldots, d_{m}\right), 1 / q_{r}=(1-\eta) / q_{r}^{0}+\eta / q_{r}^{1}$

$$
\left(\bar{A}_{\theta \vec{q}_{0}}, \bar{A}_{\theta \vec{q}_{1}}\right)_{\eta \vec{d}}=\bar{A}_{\theta \vec{q}} .
$$

Если $\theta_{0} \neq \theta_{1} \quad$ u $\theta=(1-\eta) \theta_{0}+\eta \theta_{1}$, mo

$$
\left(\bar{A}_{\theta_{0} \vec{q}_{0}}, \bar{A}_{\theta_{1} \vec{q}_{1}}\right)_{\eta \vec{d}}=\bar{A}_{\theta \vec{d}} .
$$

ТЕОРема 6 (о билинейной интерполяции). Пусть $T$ - билинейный операmop, $T:\left(A_{0}, A_{1}\right) \times\left(B_{0}, B_{1}\right) \rightarrow\left(C_{0}, C_{1}\right)$. Тогда $T: \bar{A}_{\theta p \vec{d}} \times \bar{B}_{\theta r \infty} \rightarrow \bar{C}_{\theta q \vec{d}} u$, в частности, $T: \bar{A}_{\theta p} \times \bar{B}_{\theta r \infty} \rightarrow \bar{C}_{\theta q}$. Здесь $1+1 / q=1 / p+1 / r, 1 \leqslant p, q, r \leqslant \infty$.

Автор выражает глубокую признательность В. И. Овчинникову за полезные обсуждения результатов данной работы.

\title{
ЛитературА
}

1. Lions J.-L. I-III, Ann. Scuola Norm. Sup. Pisa, 13, 389-403 (1959); Ibid, 14, 317331 (1960); J. Math. Pures Appl., 42, 195-203 (1963). 2. Lions J.-L., Peetre J. Inst. Hautes Études Sci. Publ. Math., 19, 5-68 (1964). 3. Peetre J. Lecture Notes, Brasilia, 1963 [Notas de Matematica, Vol. 39, 1968, pp. 1-68]. 4. Дмитриев В. И., Овчинников В. И. ДАН СССР, 246, №4, 794-797 (1979). 5. Брудный Ю. А., Кругляк Н. Я. ДАН СССР, 256, №1, 14-17 (1981).

Институт прикладной математики НАН Республики Казахстан

Поступило в редакцию 6 июня 1995 г. В переработанном виде 27 марта 1996 г.

УДК $512.7+514.14$

\section{Замечание о полиноме Гильберта сферического многообразия ${ }^{\star}$}

\author{
(c) 1997. А. Ю. Окуньков
}

Красивая формула для степени сферического многообразия была получена Б. Я. Казарновским [7] (в частном случае) и М. Брионом [4] (в общем случае). Эта формула обобщает теорему Кушниренко [8] (см. также, например, [11] и [1]). Для нормального сферического многообразия Брион вычислил весь полином Гильберта. Мы сформулируем эти результаты в следующей форме.

* Автор благодарен Международному научному фонду и Российскому фонду фундаментальных исследований за финансовую поддержку 
Пусть дано представление редуктивной группы $G$ в линейном пространстве $V$. Пусть $X$ - неприводимое $G$-инвариантное подмногообразие проективного пространства $\mathbb{P}(V)$. Многообразие $X$ называется сферическим, если борелевская подгруппа $B$ группы $G$ имеет на $X$ открытую орбиту.

Пусть $P$ - решетка весов группы $G$ и $L=P \otimes_{\mathbb{Z}} \mathbb{R}$ - вещественное линейное пространство, натянутое на $P$. С многообразием $X$ можно связать некоторый выпуклый многогранник $\Gamma(X) \subset L$. Для этого обозначим через $F[X]$ однородное координатное кольцо многообразия $X$; оно градуировано степенью многочленов:

$$
F[X]=\bigoplus_{n=0}^{\infty} F[X]_{n}
$$

Разложим пространство $F[X]_{n}$ на неприводимые $G$-модули:

$$
F[X]_{n}=\bigoplus_{\lambda} m_{\lambda, n} V^{\lambda}
$$

где $V^{\lambda}$ - неприводимый $G$-модуль со старшим весом $\lambda$, а $m_{\lambda, n}-$ его кратность в модуле $F[X]_{n}$. В случае сферического многообразия $X$ всегда $m_{\lambda, n} \in$ $\{0,1\}$, иными словами, спектр всегда простой. По определению [3]

$$
\Gamma(X)=\text { выпуклая оболочка }\left\{\lambda / n \mid m_{\lambda, n} \neq 0\right\} .
$$

Легко проверить [3], что это многогранник. Над комплексным полем многогранник $\Gamma(X)$ может быть эквивалентным образом определен как пересечение образа отображения момента с камерой Вейля [3] (см. также [5] и [6]).

Если многообразие $X$ нормально, то, как показал Брион,

$$
F[X]_{n}=\bigoplus_{\lambda \in n \Gamma(X) \cap P^{\prime}} V^{\lambda}
$$

где $P^{\prime}$ - некоторая подрешетка в $P$; ее определение см. в [4]. Следовательно, по формуле Г. Вейля

$$
\operatorname{dim} F[X]_{n}=\sum_{\lambda \in n \Gamma(X) \cap P^{\prime}} \operatorname{dim} V^{\lambda}=\sum_{\lambda \in n \Gamma(X) \cap P^{\prime}} \prod_{\alpha>0} \frac{(\lambda+\rho, \alpha)}{(\rho, \alpha)},
$$

где $\alpha$ пробегает положительные корни $\alpha \in P$, а $\rho$ есть полусумма положительных корней. Из этой формулы вытекает, что

$$
\operatorname{deg} X=(\operatorname{dim} X) ! \int_{\Gamma(X)} \prod_{\alpha>0,(\lambda, \alpha) \neq 0} \frac{(\lambda, \alpha)}{(\rho, \alpha)} d \lambda,
$$

где мера Лебега $d \lambda$ нормирована так, что объем ячейки решетки $P^{\prime}$ равен единице. Последняя формула верна и для многообразия $X$, не являющегося нормальным. Если группа $G$ есть тор, то подынтегральная функция превращается в единицу и получается теорема Кушниренко о степени торического многообразия

$$
\operatorname{deg} X=(\operatorname{dim} X) ! \operatorname{vol} \Gamma(X) .
$$


Наше замечание теперь состоит в том, что для любой классической группы $G$ и любого сферического $G$-многообразия $X$ можно определить в вещественном пространстве $\mathbb{R}^{\operatorname{dim} B}$ размерности $\operatorname{dim} B$ многогранник $\Delta(X)$ и решетку $\widetilde{P}$ со следующими свойствами:

ПРЕДЛОЖЕНИЕ. Если многообразие $X$ нормально, то

$$
\operatorname{dim} F[X]_{n}=\operatorname{card}\{n \Delta(X) \cap \widetilde{P}\},
$$

т.е. полином Гильберта многообразия $X$ совпадает с полиномом Эрхарта многогранника $\Delta(X)$. Для любого многообразия $X$

$$
\operatorname{deg} X=(\operatorname{dim} X) ! \operatorname{vol} \Delta(X),
$$

где объем нормирован так, что объем ячейки $\widetilde{P}$ равен единиче.

Определение многогранника $\Delta(X)$ чрезвычайно просто. Напомним, что с каждым неприводимым представлением $V^{\lambda}$ классической группы $G$ связан многогранник Гельфанда-Цетлина, который мы обозначим через $\Delta(\lambda)$. Для группы $G L(k)$ многогранник $\Delta(\lambda)$ задается неравенствами промежуточности

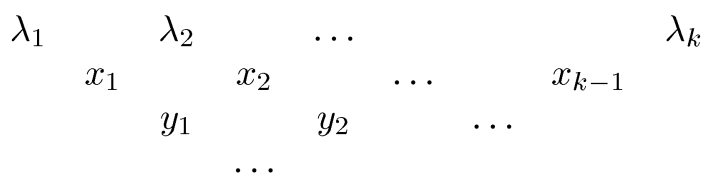

где $x_{1}, x_{2}, \ldots, y_{1}, \ldots$ суть вещественные переменные, а запись

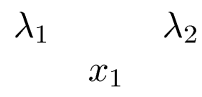

означает, что $\lambda_{1} \geqslant x_{1} \geqslant \lambda_{2}$. Определение многогранников Гельфанда-Цетлина для ортогональной и симплектической групп см. в [2]. Во всех случаях $\Delta(\lambda)$ лежит в вещественном пространстве той же размерности, что и размерность максимальной унипотентной подгруппы $N \subset G$. Для нас важно следующее свойство многогранника $\Delta(\lambda)$ :

$$
\operatorname{dim} V^{\lambda}=\text { число целых точек в } \Delta(\lambda) .
$$

Определим $\Delta(X)$ как следующее подмножество пространства $L \oplus \mathbb{R}^{\operatorname{dim} N}=$ $\mathbb{R}^{\operatorname{dim} B}$ :

$$
\Delta(X)=\bigcup_{\lambda \in \Gamma(X)}(\lambda, \Delta(\lambda))
$$

Поскольку $\Delta(\lambda)$ есть выпуклый многогранник, линейно зависящий от $\lambda \in L$, множество $\Delta(X)$ - выпуклый многогранник. Его можно рассматривать как расслоение над $\Gamma(X)$ со слоем $\Delta(\lambda)$. Наконец, положим

$$
\widetilde{P}=P^{\prime} \oplus \mathbb{Z}^{\operatorname{dim} N} \subset L \oplus \mathbb{R}^{\operatorname{dim} N} .
$$


В силу (3) равенство (2) есть простая переформулировка (1).

Определение многогранника $\Delta(X)$ можно сделать менее формальным. Можно указать процедуру построения выпуклых многогранников по любым неприводимым проективным многообразиям [9]. При этом многогранники $\Delta(\lambda)$ будут отвечать многообразиям флагов группы $G[10]$.

Я благодарен М. Бриону, В. А. Гинзбургу, Б. Я. Казарновскому, Г. И. Ольшанскому и А. Г. Хованскому за полезные обсуждения.

\title{
ЛИТЕРАТУРА
}

1. Atiyah M. F. Proc. Edinburgh Math. Soc. (2), 26, No. 2, 121-133 (1983). 2. Berenstein A., Zelevinski A. J. Geom. Phys., 5, No. 3 (1989). 3. Brion M. In: Séminaire d'Algèbre Paul Dubreil et Marie-Paul Malliavin. Lect. Notes in Math., Vol. 1296, Springer-Verlag, 1987. 4. Brion M. Duke Math. J., 58, 397-424 (1989). 5. Guillemin V., Sternberg S. Invent. Math., 67, No. 3, 515-538 (1982). 6. Heckmann G. J. Invent. Math., 67, 333-356 (1982). 7. Казарновский Б. Я. Функц. анализ и его прил., 21, вып. 4 (1987). 8. Kouchnirenko A. Invent. Math., 32, 1-31 (1976). 9. Okounkov A. Invent. Math., 125, 405-411 (1996). 10. Okounkov A. to appear in A. A. Kirillov anniversary volume, Adv. Sov. Math., Am. Math. Soc. 11. Хованский А. Г. Функц. анализ и его прил., 26, вып. 4, 57-63 (1992).

Институт проблем передачи информации РАН e-mail: okounkov@ippi.ac.msk.su

Поступило в редакцию 25 декабря 1995 г.

\section{Предельное поведение спектра в одном классе больших случайных матриц}

\author{
(c) 1997. А. Ю. ПлАхов
}

1. Уравнение Марченко-Пастура. В статье В. А. Марченко и Л. А. Пастура [1] рассматривается случайный самосопряженный оператор в $\mathbb{C}^{N}$ вида $A_{N}=A_{0 N}+\sum_{n=1}^{m} \tau_{n}\left(\cdot, q^{n}\right) q^{n}$, где $\tau_{n}$ - действительные независимые случайные величины с одинаковой функцией распределения $\sigma(\tau)$, случайные векторы $q^{n}$ независимы и удовлетворяют некоторым дополнительным условиям, a оператор $A_{0 N}$ неслучаен. Далее, с помощью формулы $\nu_{N}(\lambda)=\{$ число собственных значений $A_{N}$, меньших, чем $\left.\lambda\right\} / N$ определяется нормированная спектральная функция оператора $A_{N}$. Марченко и Пастур доказали, что если (i) $\lim _{N \rightarrow \infty}(m / N)=c$ и (ii) последовательность нормированных спектральных функций операторов $A_{0 N}$ сходится к некоторой монотонно неубывающей функции $\nu_{0}(\lambda)$ во всех ее точках непрерывности, то $\nu_{N}(\lambda)$ сходится по вероятности к неслучайной неубывающей функции $\nu(\lambda)$ во всех ее точках непрерывности и преобразование Стилтьеса этой функщии, $u(z)=\int_{-\infty}^{+\infty}(\lambda-z)^{-1} d \nu(\lambda)$, может быть найдено следующим образом.

* Эта работа выполнена при частичной поддержке фондов INTAS, грант RFBR 95418, RFFI-DFG, грант 96-01-000436. 